\title{
CALIFA reveals prolate rotation in massive early-type galaxies: A polar galaxy merger origin?
}

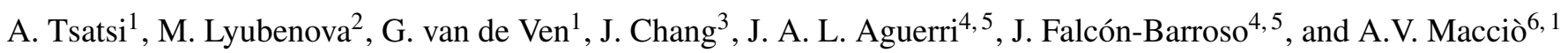 \\ 1 Max-Planck-Institut für Astronomie, Königstuhl 17, 69117 Heidelberg, Germany \\ e-mail: tsatsi@mpia.de \\ 2 Kapteyn Astronomical Institute, University of Groningen, Postbus 800, 9700 AV Groningen, The Netherlands \\ ${ }^{3}$ Purple Mountain Observatory, the Partner Group of the MPI für Astronomie, 2 West Beijing Road, Nanjing 210008, PR China \\ 4 Instituto de Astrofísica de Canarias, vía Láctea s/n, 38205 La Laguna, Tenerife, Spain \\ 5 Departamento de Astrofísica, Universidad de La Laguna, 38205 La Laguna, Tenerife, Spain \\ 6 New York University Abu Dhabi, PO Box 129188, Saadiyat Island, Abu Dhabi, UAE
}

Received 8 December 2016 / Accepted 12 July 2017

\section{ABSTRACT}

\begin{abstract}
We present new evidence for eight early-type galaxies (ETGs) from the CALIFA Survey that show clear rotation around their major photometric axis ("prolate rotation"). These are LSBCF560-04, NGC 0647, NGC 0810, NGC 2484, NGC 4874, NGC 5216, NGC 6173, and NGC 6338. Including NGC 5485, a known case of an ETG with stellar prolate rotation, as well as UGC 10695, a further candidate for prolate rotation, we report ten CALIFA galaxies in total that show evidence for such a feature in their stellar kinematics. Prolate rotators correspond to $\sim 9 \%$ of the volume-corrected sample of CALIFA ETGs, a fraction much higher than previously reported. We find that prolate rotation is more common $(\sim 27 \%)$ among the most massive ETGs $\left(M_{*} \gtrsim 2 \times 10^{11} M_{\odot}\right)$. We investigated the implications of these findings by studying $N$-body merger simulations, and we show that a prolate ETG with rotation around its major axis could be the result of a major polar merger, with the amplitude of prolate rotation depending on the initial bulgeto-total stellar mass ratio of its progenitor galaxies. Additionally, we find that prolate ETGs resulting from this formation scenario show a correlation between their stellar line-of-sight velocity and higher order moment $h_{3}$, opposite to typical oblate ETGs, as well as a double peak of their stellar velocity dispersion along their minor axis. Finally, we investigated the origin of prolate rotation in polar galaxy merger remnants. Our findings suggest that prolate rotation in massive ETGs might be more common than previously expected, and can help toward a better understanding of their dynamical structure and formation origin.
\end{abstract}

Key words. galaxies: elliptical and lenticular, $\mathrm{cD}$ - galaxies: formation - galaxies: kinematics and dynamics - galaxies: structure galaxies: stellar content

\section{Introduction}

More than 60 years have passed since Contopoulos (1956) suggested that the intrinsic shape of elliptical galaxies could be triaxial. Since then, it has been established that the existence and persistence of triaxial galaxies is theoretically permitted (e.g. Aarseth \& Binney 1978; Binney 1985), and that if elliptical, or in general, early-type galaxies (ETGs), are such systems, then they are expected to show two types of stable stellar rotation: rotation around their short axis, as in the typical case of oblate systems (oblate rotation), and rotation around their long axis, socalled "prolate rotation". We note that "prolate rotation" is often referred to as "long-axis" rotation, or "minor-axis" rotation, the latter meaning a velocity gradient along their projected minor axis.

This suggests that any observations of an undisturbed earlytype galaxy that shows rotation around its major apparent axis is indicative of its triaxial shape. However, several attempts to find such systems have been unsuccessful in the past (e.g., Bertola et al. 1988), and until now, only a few observations of galaxies with clear prolate rotation exist. These cases are NGC 1052 (Schechter \& Gunn 1979; Davies \& Illingworth 1986), NGC 4406, NGC 5982, NGC 7052, NGC 4365, NGC 5485 (Wagner et al. 1988), NGC 4261 (Davies \& Illingworth 1986; Wagner et al. 1988), NGC 4589 (Wagner et al. 1988; Moellenhoff \& Bender 1989), AM 0609-331 (Moellenhoff \& Marenbach 1986), M 87 (Davies \& Birkinshaw 1988; Emsellem et al. 2014), NGC 4473 (Foster et al. 2013), and NGC 5557 in Krajnović et al. (2011), totalling 12 objects. There is also evidence for (although not clear) prolate rotation in NGC 2749, IC 179 (Jedrzejewski \& Schechter 1989), NGC 3923 (Carter et al. 1998) and NGC 7626 (Davies \& Birkinshaw 1988).

Most of these prolate rotators belong in the potential wells of galaxy groups or clusters, while 3 of them, NGC 4589, NGC 5485, and AM 0609-331, show strong dust lanes along their minor axes, providing an additional visual evidence of triaxiality. Only for 6 of the above 12 known prolate rotating ETGs, two-dimensional integral field unit (IFU) spectroscopy of the stellar kinematics has been carried out so far: NGC 4261, NGC 5485, NGC 4365, NGC 4406, NGC 5557, and M 87 (Davies et al. 2001; Emsellem et al. 2004; Krajnović et al. 2011; Emsellem et al. 2014). These studies have revealed that prolate rotation may often coexist with oblate rotation, often in the form of a kinematically decoupled component (KDC), a central stellar component with distinct kinematic properties from those of the main body of the galaxy, as is often found to reside in many ETGs (e.g., McDermid et al. 2006; Krajnović et al. 2011).

While the dynamical stability of prolate rotation in triaxial galaxies has been extensively studied theoretically, constraining the formation origin of such systems is yet a highly challenging task, mainly because only few observations of prolate-rotating ETGs exist in the literature.

A growing amount of evidence from cosmological simulations suggests that massive ETGs of stellar mass $M_{*} \gtrsim$ $10^{11} M_{\odot}$ have been assembled in two phases: (i) an early rapid 
dissipational formation phase (such as a gas-rich major merger), taking place at redshift $z>2$ and contributing to the main buildup of the central 1-2 effective radii $\left(r_{\mathrm{e}}\right)$ of the galaxy, followed by a (ii) second phase of satellite accretion (gas-poor minor mergers) that built up their outer parts $\left(r>1-2 r_{\mathrm{e}}\right)$ at $z<2$ (e.g., Vitvitska et al. 2002; Khochfar \& Silk 2009; Johansson et al. 2012; Lackner et al. 2012; Naab et al. 2014). This "two-phase" assembly formation scenario is in line with a series of observational lookback studies that report a significant growth in mass and in size of massive ETGs at radii $r>1-2 r_{\mathrm{e}}$ since $z \sim 2$ (e.g., Zirm et al. 2007; van Dokkum et al. 2008, 2010; Pérez et al. 2013; Burke \& Collins 2013; van der Wel et al. 2014). It is also supported by observations of the ETG luminosity function evolution (e.g., Bell et al. 2004; Faber et al. 2007), as well as widefield observations of the stellar kinematics of many ETGs that show central oblate rotating components within $1-2 r_{\mathrm{e}}$, consistent with being formed through an initial gas-rich major merger, embedded in more spherical and slowly rotating structures that dominate at larger radii $\left(2-4 r_{\mathrm{e}}\right)$, consistent with a late satellite accretion assembly phase (Arnold et al. 2014).

However, in the case of ETGs that show strong prolate rotation in their central regions $\left(r<1-2 r_{\mathrm{e}}\right)$, it is not clear whether and how the above picture of an early and rapid dissipational process could form a triaxial inner structure with no (significant) oblate rotation. It is possible that the two-phase assembly scenario, where the central region was formed by a major merger, still holds for this special case of prolate rotators. This is supported by the study of Naab \& Burkert (2003), Jesseit et al. (2007), who showed that the prolate rotation in the remnant of a 1:1 merger is stronger than in unequal-mass merger remnants. Cox et al. (2006) showed, however, that dissipational equal-mass disk mergers result in merger remnants with stronger oblate rotation. Bois et al. (2011) compared the kinematics of merger remnants with the observed kinematics of ETGs and also showed that slow rotators formed in 1:1 mergers can show significant kinematic misalignments, with strong prolate rotation in the case of gas-poor merger remnants (Hoffman et al. 2010).

Given the motivations above, it is most likely that the inner regions of present-day massive ETGs that show strong prolate rotation and no (significant) oblate rotation may have been formed preferentially by gas-poor (dry) major mergers. However, it is not yet clear why only a few observations of such systems exist in the literature so far.

Motivated by all the above evidence, we have made use of the Calar Alto Legacy Integral Field Area (CALIFA) survey (Sánchez et al. 2012), which provides IFU data for a statistically well-defined sample of $\sim 600$ galaxies across the Hubble sequence, in order to search for possible prolate rotating ETGs. Here, we present ten massive $\left(M_{*} \sim 10^{11} M_{\odot}\right)$ ETGs that show prolate rotation in their stellar kinematics, including one known galaxy (NGC 5485), the discovery of eight new clear cases of massive prolate-rotating ETGs, and one further candidate (UGC 10695), adding a significant fraction to the cases of prolate rotation that exist in the literature so far.

Additionally, we investigate a possible merger origin of these systems by studying the kinematics of simulated ETGs formed in $N$-body simulations of major mergers in an observational-like fashion. We show that polar major mergers of disk galaxies can produce prolate-shaped merger remnants with strong rotation around their major axis, which could be the progenitors of the observed present-day massive prolate rotating ETGs. Such a formation scenario is in line with the existing picture of a twophase assembly of massive ETGs and can explain the rarity of observations of prolate rotators as a natural consequence of the infrequency of dry major polar mergers.

This paper is organized as follows: in Sect. 2 we describe the sample and present the observations of prolate rotators in the CALIFA Survey, in Sect. 3 we describe the set of major merger simulations, the resulting shape and kinematics of the simulated remnant ETGs, and the origin of their prolate rotation. Finally, we conclude in Sect. 4.

\section{Observations}

The Calar Alto Legacy Integral Field Area (CALIFA) survey (Sánchez et al. 2012) provides IFU data for a statistically welldefined sample of $\sim 600$ galaxies across the Hubble sequence. The CALIFA sample is selected from a mother sample of 937 galaxies in the photometric catalogue of the 7th data release of the Sloan Digital Sky Survey (SDSS; Abazajian et al. 2009), with the main selection criteria being an angular isophotal diameter of $45^{\prime \prime} \leq D_{25} \leq 80^{\prime \prime}$ and a redshift range of $0.005 \leq z \leq 0.03$.

The survey uses the PPAK IFU (Verheijen et al. 2004; Kelz et al. 2006) of the Potsdam Multi-Aperture Spectrograph, PMAS (Roth et al. 2005) at the $3.5 \mathrm{~m}$ telescope of CAHA, with a field of view that can extend up to several effective radii $\left(r_{\mathrm{e}}\right)$, using two different setups (V500 and V1200) of resolutions, $R \sim 850$ and $R \sim 1650$. For a more detailed description of the CALIFA survey and extraction of stellar kinematics, see Walcher et al. (2014), Falcón-Barroso et al. (2017).

In this work we focus on observations of 10 ETGs, chosen by visual inspection of the stellar kinematics of the CALIFA 3rd data release (DR3) sample (Sánchez et al. 2016) of 667 galaxies. These are LSBCF560-04, NGC 0647, NGC 0810, NGC 2484, NGC 4874, NGC 5216, NGC 5485, NGC 6173, NGC 6338, and UGC 10695. Of these 10 galaxies, 7 belong to the CALIFA Kinematic Subsample of 81 ETGs (E+S0s) described in Falcón-Barroso et al. (2017), a statistically well-defined sample of $\sim 300$ galaxies in total, while 3 (LSBCF560-04, NGC 0647, and NGC 2484) belong to the CALIFA Extension Sample, an inhomogeneous sample of galaxies observed in the CALIFA setup (Sánchez et al. 2016). Some of their qualitative properties, including their Hubble types, group memberships, distances, stellar masses, and effective radii, are shown in Table 1.

We derived our stellar kinematics maps following the strategy described in Falcón-Barroso et al. (2017). However, we adopted a few differences. We used the CALIFA V500 data set alone, as these observations typically reach fainter magnitudes, and in this way, we are able to better recover the stellar kinematics in the outer parts of the galaxies. In order to reliably measure the line-of-sight velocity, velocity dispersion, and the higher order Gauss-Hermite terms $h_{3}$ and $h_{4}$, we binned the data to $S / N \sim 40$ using the Voronoi binning technique as implemented by Cappellari \& Copin (2003). Then we used a subset of 330 stars from the IndoUS library (Valdes et al. 2004, the same set as in Falcón-Barroso et al. 2017), and fit the binned spectra in the wavelength range 4250.0-5500.0 A.

In this way, we extracted the stellar kinematics shown in Fig. 1. The CALIFA maps reveal prolate rotation for all ten galaxies, with amplitudes ranging from $\sim 40$ to $100 \mathrm{~km} \mathrm{~s}^{-1}$. In all cases, we find strong kinematic evidence for triaxiality, as the stellar rotation of the main body of the galaxy is prolate (around the major apparent axis). In the case of UGC 10695, there is evidence for prolate rotation in its stellar line-of-sight velocity 
Table 1. Properties of the CALIFA prolate rotators.

\begin{tabular}{llcccc}
\hline \hline Name & T/G & $\begin{array}{c}d \\
(\mathrm{Mpc})\end{array}$ & $\begin{array}{c}M_{*} \\
\left(10^{11} M_{\odot}\right)\end{array}$ & $\begin{array}{c}r_{\mathrm{e}} \\
(\mathrm{kpc})\end{array}$ & $\begin{array}{c}\Psi \\
(\mathrm{deg})\end{array}$ \\
\hline LSBCF560-04 & E5/BCG & 238 & $7.1 \pm 0.6^{a}$ & 17.9 & $57 \pm 5$ \\
NGC 0647 & E7/in a group & 184 & $3.7 \pm 0.3^{a}$ & 8.7 & $72 \pm 3$ \\
NGC 0810 & E5/in a pair & 110 & $2.12_{-0.08}^{+0.16 b}$ & 9.3 & $87 \pm 2$ \\
NGC 2484 & E4/BCG & 192 & $5.0 \pm 0.5^{a}$ & 12.5 & $52 \pm 4$ \\
NGC 4874 & E0/BCG & 102 & $2.8_{-1.1}^{+1.6 b}$ & 12.4 & $86 \pm 5$ \\
NGC 5216 & E0/in a pair & 42 & $0.230_{-0.020}^{+0.02 b}$ & 4.1 & $66 \pm 5$ \\
NGC 5485 & E5/in a group & 27 & $0.9_{-0.4}^{+0.1 b}$ & 4.1 & $80 \pm 3$ \\
NGC 6173 & E6/BCG & 126 & $2.5_{-0.2}^{+0.3 b}$ & 30.5 & $80 \pm 2$ \\
NGC 6338 & E5/BCG & 117 & $3.0_{-0.7}^{+0.3 b}$ & 17.0 & $36 \pm 4$ \\
UGC 10695 & E5/in a group & 120 & $2.7 \pm 0.2^{a}$ & 15.7 & $87 \pm 2$ \\
\hline
\end{tabular}

Notes. Column 1: name. Column 2: Hubble type T, as in Walcher et al. (2014), and group membership G (NED/SIMBAD). Column 3: redshiftbased distance in Mpc. Column 4: available stellar mass estimates, ${ }^{(a)}$ from WISE photometry by Norris et al. (2016), and ${ }^{(b)}$ from CALIFA DR3, as in Walcher et al. (2014). Column 5: effective radius, determined by growth-curve analysis of SDSS images of each galaxy, as in Walcher et al. (2014). Column 6: global kinematic misalignment angle $\Psi$. Cosmological angular size distances are calculated by adopting $H_{0}=70 \mathrm{~km} \mathrm{~s}^{-1} \mathrm{Mpc}^{-1}$, $\Omega_{\mathrm{m}}=0.3, \Omega_{\Lambda}=0.7{ }^{(*)} \mathrm{UGC} 10695$ is listed as a further candidate, showing evidence for prolate rotation.

map (not as clear as in the other cases, however), and it is therefore listed as a further candidate in our sample. In the case of NGC 6338, the rotation is prolate only in its inner parts, while the outer parts show oblate rotation - new evidence of an elliptical galaxy with a kinematically decoupled prolate-rotating component.

In order to quantify the kinematic misalignment for our sample of galaxies, we estimated the global kinematic misalignment angle $\Psi$ as defined in Franx et al. (1991):

$\sin \Psi=\left|\sin \left(\mathrm{PA}_{\text {phot }}-\mathrm{PA}_{\text {kin }}\right)\right|$,

where $\mathrm{PA}_{\text {phot }}$ is the position angle of the photometric major axis, measured in the outer parts of each galaxy using SDSS images, measured as in Walcher et al. (2014), while $\mathrm{PA}_{\text {kin }}$ is the global kinematic position angle measured from the CALIFA velocity maps of each galaxy, as in Krajnović et al. (2006, see Appendix C). Angle $\Psi$ as defined above shows the projected misalignment between photometry and kinematics of a stellar system. Typically, values of $\Psi<25^{\circ}$ describe a regular rotator (e.g. Kutdemir et al. 2008). For most ETGs, this quantity is rather low, showing alignment typical for oblate systems, and for example, in the ATLAS ${ }^{3 D}$ Survey, approximately $90 \%$ of ETGs show $\Psi<15^{\circ}$ (Krajnović et al. 2011).

Table 1 shows the derived global misalignment angles for all the galaxies in our sample. For most galaxies, we find $\Psi>$ $50^{\circ}$. For NGC 6338, we find $\Psi=36^{\circ} \pm 4^{\circ}$, as there is prolate rotation in the inner parts, while the apparent oblate rotation of the galaxy in the outer parts influences the value of global $\Psi$. UGC 10695 , listed here as a candidate, shows $\Psi=87^{\circ} \pm 2^{\circ}$, a further indication for prolate rotation.

It is interesting to note that almost all of the galaxies in our sample belong in groups or clusters. Five out of ten are the brightest galaxies in their clusters (BCGs), with relatively high stellar masses $\left(M_{*} \gtrsim 2 \times 10^{11} M_{\odot}\right)$.

Moreover, two galaxies in our sample show strong dust lanes along their minor photometric axis in their SDSS images (Fig. 1): NGC 5485, a known case of an ETG with prolate rotation and a minor-axis dust lane (Wagner et al. 1988; Emsellem et al. 2011), and the new case of NGC 0810. Assuming that dust and gas settle in the principal planes of a galaxy, the existence of a minor-axis dust lane is visual evidence for triaxiality (Bertola \& Galletta 1978; Merritt \& de Zeeuw 1983). We expect that the ten prolate rotators of our sample must be to some extent triaxial or prolate.

We find that prolate rotation, and the triaxiality in massive ETGs implied from this stellar kinematic feature, may not be as rare as previously thought. In order to estimate the occurrence rate of this kinematic feature in ETGs, we considered the subset of 6 prolate rotators that belong to the statistically well-defined CALIFA Kinematic Subsample of 81 ETGs (Falcón-Barroso et al. 2017) ${ }^{1}$.

After applying volume corrections for each galaxy, following Walcher et al. (2014), we report a fraction of 9\% of prolaterotating ETGs in the volume of the CALIFA Kinematic Subsample ETGs. Notably, this fraction becomes $\sim 27 \%$ in the volume of ETGs with stellar masses $M_{*} \gtrsim 2 \times 10^{11} M_{\odot}$.

These fractions seem to agree with the corresponding ones from the ATLAS ${ }^{3 \mathrm{D}}$ Survey, where 6 galaxies (NGC 4261, NGC 4365, NGC 4406, NGC 5485, NGC 5557, and M 87) were identified to show prolate rotation in their stellar kinematics. This means that 6 out of 51 ETGs have prolate rotation in the mass range of ETGs with $M_{*} \gtrsim 10^{11} M_{\odot}$, a fraction of $\sim 12 \%$, which becomes $\sim 23 \%$ (5 out of 22 ) in the mass range of $M_{*} \gtrsim 2 \times 10^{11} M_{\odot}$ of ATLAS $^{3 \mathrm{D}}$ (D. Krajnović, E. Emsellem; priv. comm., Krajnović et al. 2011; Emsellem et al. 2014).

What are the implications of these findings for the formation of such systems? Could prolate rotators be the end-products of major mergers? Considering the rarity of observations of such systems so far, their formation origin is still poorly understood. According to the two-phase assembly scenario, the central parts of massive ETGs have been preferentially formed through gasrich major mergers approximately $\sim 10 \mathrm{Gyr}$ ago. However we do not observe clear signs of oblate rotation in most of the CALIFA prolate rotators presented here. This suggests that for this special type of objects their merger origin might have been rather gas-poor. In what follows, we explore the dynamical structure of remnants resulting from such a formation scenario.

1 UGC 10695 is listed as a prolate rotator candidate and as such, is not included in this fraction. We have also excluded the rest of the galaxies from our sample (LSBCF560-04, NGC 0647 and NGC 2484), which belong to the inhomogeneous collection of the CALIFA Extension Sample, and for which volume corrections cannot be applied. 



Fig. 1. CALIFA sample of prolate rotators. Top panel: LSBCF560-04, NGC 0647, NGC 0810, NGC 2484, and NGC 4874. The top row shows a color-composite SDSS image of each galaxy, while the second and third rows show the stellar line-of-sight velocity $V$ and the velocity dispersion $\sigma$ in $\mathrm{km} \mathrm{s}^{-1}$, respectively, extracted from the V500 CALIFA dataset. The dashed line shows the photometric major axis, measured in the outer parts of each galaxy, using SDSS images. The dotted line shows the global kinematic axis, measured as in Krajnović et al. (2006, see Appendix C). Bottom panel: same as top panel for NGC 5216, NGC 5485, NGC 6173, NGC 6338, and UGC 10695.

\section{Polar galaxy mergers}

We present a possible formation origin of prolate rotation in massive ETGs by perfoming $N$-body simulations of major mergers of spiral galaxies in polar orbits.

\subsection{Simulations}

The binary merger simulations we used were performed using the TreeSPH-code GADGET-3 (see Springel 2005, for a detailed description of the previous version of this code, GADGET-2), as used in Chang et al. (2013). In order to investigate the gaspoor merger scenario of prolate galaxies, our simulations did not include cold or hot gas (hence our simulations are $N$-body).

The two progenitor disk galaxies were identical and were composed of a stellar disk and a stellar bulge, which were embedded in a dark matter halo. The disk component followed an exponential profile, while the stellar bulge and the dark matter halo of each progenitor followed a Hernquist (1990) profile, the same as described in Springel et al. (2005). The disk of one of 
Table 2. Properties of the simulated remnants: name, mass ratio between bulge and total mass of each progenitor $B / T$, half-mass radius $r_{\mathrm{hm}}$, axial ratios $b / a, c / a$, and triaxiality parameter $T$ (see Eq. (2)) of the simulated remnants, estimated within $r_{\mathrm{hm}}$.

\begin{tabular}{cccccc}
\hline \hline Name & Progenitor $B / T$ & $\begin{array}{c}r_{\mathrm{hm}} \\
(\mathrm{kpc})\end{array}$ & $b / a$ & $c / a$ & $T$ \\
\hline M0 & 0.00 & 16.00 & 0.57 & 0.51 & 0.91 \\
M1 & 0.10 & 14.83 & 0.57 & 0.47 & 0.87 \\
M2 & 0.30 & 12.42 & 0.61 & 0.51 & 0.84 \\
M3 & 0.50 & 9.90 & 0.63 & 0.50 & 0.81 \\
\hline
\end{tabular}

the progenitor galaxies was aligned with respect to the orbital plane, while the other progenitor disk was inclined by $90^{\circ}$ with respect to its companion and the orbital plane. The initial radial and tangential velocity of each progenitor were $\sim 360$ and $180 \mathrm{~km} \mathrm{~s}^{-1}$, respectively, and their initial distance was $325 \mathrm{kpc}$.

We ran four realizations of the simulation described above, with different bulge-to-total stellar mass ratios $(B / T)$ of the progenitor galaxies. We adopted a range of $B / T$ ratios of $0.0,0.1$, 0.3 , and 0.5 (see Table 2). All the simulations had the same total number of stellar and dark matter particles $(N=2425432)$, so that each merger remnant has a stellar mass of $M_{*}=2.6 \times$ $10^{11} M_{\odot}$ and a dark matter halo of $M_{\mathrm{dm}}=1.6 \times 10^{13} M_{\odot}$. The softening length was $70 \mathrm{pc}$ for stellar and $300 \mathrm{pc}$ for dark matter particles. Each progenitor galaxy initially evolved in isolation for $2 \mathrm{Gyr}$, so that it exhibited a reasonably steady structure before the merger. After this, the merger simulation started and lasted for approximately 8 Gyr. Figure 2 shows the relative distance of the two progenitors, as well as their orbital angular momentum as a function of time during each merger simulation.

\subsection{Extracting mock observational data}

In order to connect the intrinsic mass and orbital distribution of our simulated merger remnant galaxies with observable properties, we created two-dimensional mock stellar mass and stellar kinematic maps following the method described in Tsatsi et al. (2015).

Stellar particles were projected along a chosen viewing angle and then binned on a regular grid centered on the baryonic center of mass of the galaxy. We adopted a grid size of $20 \times 20 \mathrm{kpc}^{2}$ and a pixel size of $0.5 \mathrm{kpc}$, which corresponds to the spatial resolution of CALIFA $\left(\sim 1^{\prime \prime}\right)$, assuming that our simulated galaxies were observed at a distance of $\sim 100 \mathrm{Mpc}$. The size of the corresponding "CALIFA-like" field of view extends to $\sim 1$ or 2 half-mass radii of each remnant.

The bulk velocity of the galaxy was estimated within a sphere of $50 \mathrm{kpc}$ around the center and subtracted from all particle velocities. We then extracted stellar mass-weighted lineof-sight kinematic maps for each of our merger remnants. The maps were spatially binned using the two-dimensional Voronoi binning method (Cappellari \& Copin 2003), based on a minimum number of particles per pixel in the map. The signal corresponded to the number of particles per pixel, and we adopted Poisson noise, such that our signal-to-noise ratio $(\mathrm{S} / \mathrm{N})$ per bin corresponded approximately to an average target value of $S / N \sim 35$ for all the remnants.

The mass-weighted stellar line-of-sight velocity distribution (LOSVD) was then extracted for every Voronoi bin and fit with the Gauss-Hermite series (van der Marel \& Franx 1993), as implemented by van de Ven et al. (2006, see the Appendix)

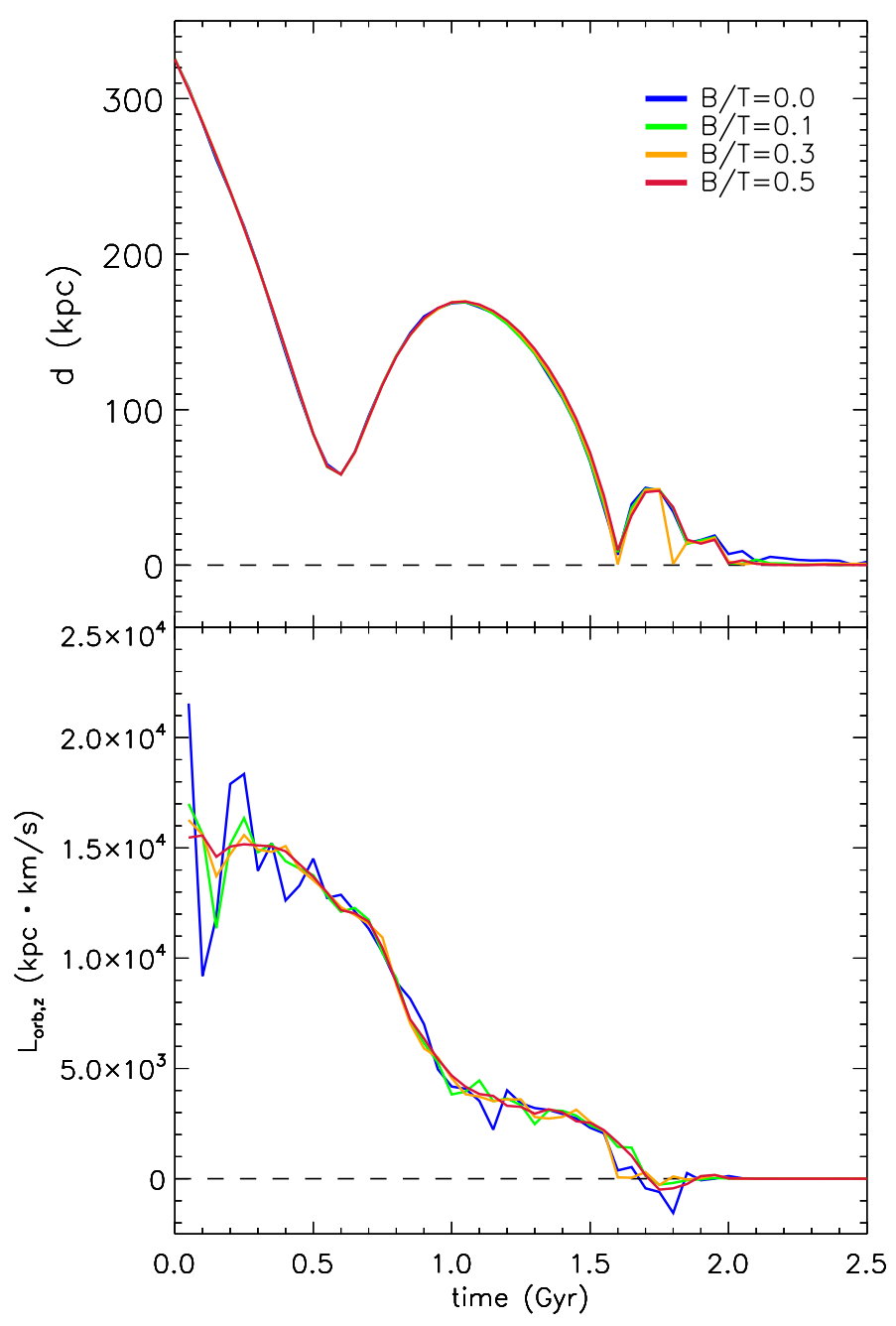

Fig. 2. Relative distance $d$ of the two progenitors (top) and orbital angular momentum $L_{\mathrm{orb}, z}$ of one of the progenitors (bottom) as a function of time during each merger simulation. Different colors correspond to the different $B / T$ ratios of each progenitor.

allowing us to retrieve the Gauss-Hermite parameters of the $\operatorname{LOSVD}\left(V, \sigma, h_{3}, h_{4}\right)$ of the final merger remnants.

\subsection{Shape and kinematics of the simulated remnants}

The shape properties of the resulting merger remnants are shown in Table 2. The remnants become more compact as the $B / T$ of their progenitors increases and their half-mass radius $\left(r_{\mathrm{hm}}\right)$ decreases.

Figure 3 shows the triaxiality parameter $T$ as a function of distance from the center of each remnant, defined as

$T=\frac{1-(b / a)^{2}}{1-(c / a)^{2}}$,

where $a>b>c$ correspond to the principal axes of an ellipsoid, computed by extracting the eigenvalues of the moment of inertia tensor within spherical shells of radius $r$ for each remnant (for oblate ellipsoids $T=0$, and for prolate ellipsoids $T=1$ ).

All our simulated remnants are highly prolate within two half-mass radii, with $T>0.8$. For radii $r>0.5 r_{\mathrm{hm}}$ there appears to be a trend of lower $T$ with increasing $B / T$ ratio of the progenitors.

The projected stellar mass, mean velocity, and velocity dispersion of all the final merger remnants are shown in Fig. 4. 




Fig. 3. Triaxiality parameter $T$ as a function of distance from the center of each remnant $r / r_{\mathrm{hm}}$ for all the simulated merger remnants. Different colors correspond to the different $B / T$ ratios of their progenitors.

All the remnants show prolate rotation. The amplitude of prolate rotation depends on the initial $B / T$ ratio of their progenitors. A lower $B / T$ ratio of the progenitors results in remnants with stronger prolate rotation, with a maximum amplitude of $\sim 100 \mathrm{~km} \mathrm{~s}^{-1}$ in the case of two bulgeless progenitors $(B / T=0)$.

The velocity dispersion shows two peaks along the minor photometric axis and a central dip for the bulgeless case. As the progenitor $B / T$ ratio increases, the double peaks become weaker. Nonetheless, the spatial resolution of the CALIFA velocity dispersion maps shown in Fig. 1 is too low and the uncertainties are relatively large, so that it is difficult to identify the existence of such substructures.

We additionally investigated the spatial structure of the higher order moments, $h_{3}$ and $h_{4}$, which are comparable to the skewness and the kurtosis of the LOSVD, respectively. Krajnović et al. (2008) found that ETGs show two different trends in their higher order kinematics: the first trend shows ETGs with a strong anticorrelation of $h_{3}$ and $V / \sigma$ with high values of $V / \sigma$, characteristic of a tail of low-velocity material in the LOSVD, and indicates the existence of a rotating disk component in the galaxy. The second trend shows an $h_{3}$ that is close to zero, which may show even positive correlation at intermediate $V / \sigma$. This is considered the result of prolate rotation (minor-axis contamination).

Figure 5 shows that for all our simulated remnants $h_{3}$ and $V / \sigma$ show a positive correlation at low $V / \sigma$ values, especially for the remnants of bulge-dominated progenitors $(B / T>0.3)$. For the remnants of more disky progenitors $(B / T<0.3)$, the distribution of $h_{3}$ and $V / \sigma$ shows a positive correlation at intermediate $V / \sigma$ and anticorrelating tails at higher $V / \sigma$ values $(V / \sigma>0.3)$.

A positive correlation between $h_{3}$ and $V / \sigma$ is indicative of a tail of high-velocity material in the LOSVD. In triaxial ellipsoids, this has been shown to be caused by the superposition of box orbits and minor-axis tube orbits in simulations of collisionless merger remnants (Bendo \& Barnes 2000; Jesseit et al. 2007). In line with these findings, we confirm this feature in our triaxial and collisionless merger remnants, and we additionally suggest that the amount of box versus minor-axis tube orbits in the final remnant seem to be linked to the orbital structure of each progenitor, as the progenitor $B / T$ ratio influences the resulting $h_{3}$ and $V / \sigma$ distribution of each remnant (Fig. 5).

Similarly, the CALIFA data are a challenge when we aim to measure the higher order moments $h_{3}$ and $h_{4}$ reliably, but we report evidence for an $h_{3}-V / \sigma$ correlation for LSBCF560-04 (Fig. 5). By calculating the Pearson correlation coefficients $\rho$ of 10000 bootstrap realizations of $h_{3}$ and $V / \sigma$ with replacement, we find that the distribution of $\rho$ resembles a Gaussian distribution, with a mean of $\bar{\rho} \times 0.26$ and $\sigma=0.14$, suggesting a weak positive correlation of $h_{3}$ and $V / \sigma$ for LSBCF560-04, which becomes stronger $(\bar{\rho}=0.50, \sigma=0.20)$ at values $V / \sigma>0.08$. With higher sensitivity and better resolution instruments, such as MUSE (Bacon et al. 2010), it will be possible and very interesting to determine whether the prolate-rotating CALIFA galaxies reported here show similar signatures in their stellar velocity dispersion and their higher-order moments as the galaxies that result from the gas-poor merger formation history assumed here.

We also find that the triaxial merger remnants exhibit figure rotation on the orbital plane. The direction of the figure rotation is the same as the orbital direction of the merger. However, this tumbling motion is very slow, and for the simulation M3, for instance, the long axis of the remnant completes one full rotation in approximately 4.5 Gyr. This figure rotation is caused either by torques exerted from the triaxial dark matter halo, or/and by angular momentum transfer from the stellar component that is expelled during the merger and is subsequently reaccreted onto the main stellar halo. Although it is beyond the scope of this paper to study the effect of figure rotation in detail, we note that if it is strong enough, it may alter the orbital structure of a galaxy and its observed kinematics (e.g., Heisler et al. 1982; van Albada et al. 1982; Wilkinson \& James 1982; Statler et al. 2004; Deibel et al. 2011). In our case, the figure rotation is slow and not evident in our line-of-sight mock kinematic maps, as each remnant is projected with the orbital plane (which is also the plane of figure rotation) viewed face-on.

\subsection{Origin of prolate rotation}

In order to understand the origin of prolate rotation better, we separated the LOSVD of the stellar particles in each remnant according to their formation origin by selecting the particles that initially formed the disks and the bulges of the two progenitors.

Figure 6 shows the remnant of two bulgeless progenitors M0 and the remnant of two bulge-dominated $(B / T=0.5)$ progenitors M3. The particles that initially formed the disk of the progenitor that was inclined by $90^{\circ}$ with respect to the orbital plane of the merger ("Polar Pr. disk") show strong prolate rotation in the final merger remnant, with an amplitude of $\sim 200 \mathrm{~km} \mathrm{~s}^{-1}$ for both simulation setups. The particles that initially formed the disk of the progenitor whose disk was aligned with the orbital plane ("Aligned Pr. disk") show very weak rotation with an amplitude of $\sim 10 \mathrm{~km} \mathrm{~s}^{-1}$. The remaining stellar particles that initially formed the bulges of the two progenitors ("Pr. bulges") show no rotation in the final remnant (M3).

This implies that depending on the $B / T$ of the progenitors, the stellar population that accounts mainly for the prolate rotation may contribute significantly $(\sim 50 \%)$ to the total mass of a remnant of disky progenitors (M0), while in the case of two bulge-dominated progenitors (M3), this component may be only $\sim 25 \%$ of the total mass of the remnant.

The latter seems to be in agreement with findings for NGC 4365, a well-studied elliptical galaxy that shows prolate rotation in its outer parts with an amplitude of $\sim 60 \mathrm{~km} \mathrm{~s}^{-1}$ (Davies et al. 2001). By constructing triaxial Schwarzschild dynamical models, van den Bosch et al. (2008) have shown that the stellar component of this galaxy that accounts for its prolate rotation shows an amplitude of stellar rotation that is larger than 


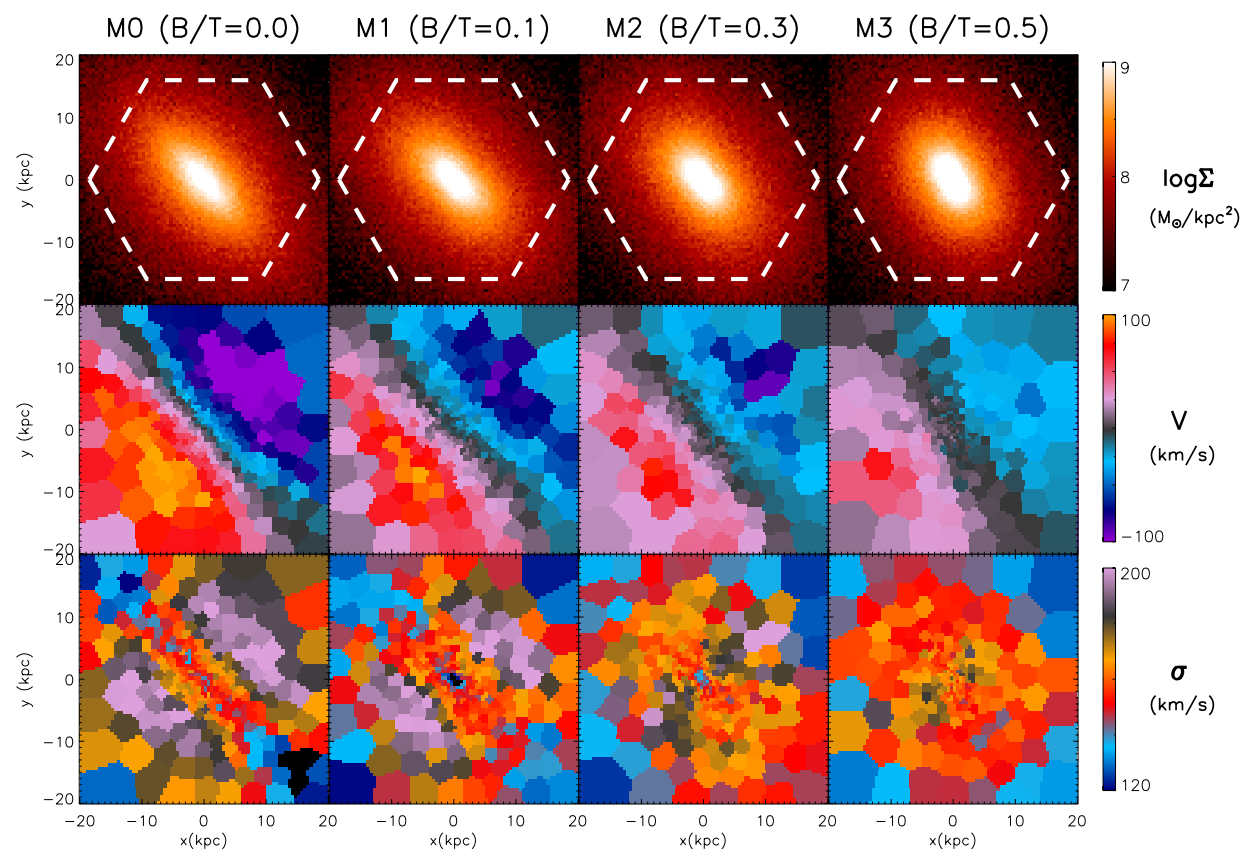

Fig. 4. Stellar mass and line-of-sight orbital distribution of the simulated merger remnants M0-3 (the $B / T$ ratio of their progenitors increases from left to right). The projected plane $x-y$ corresponds to the orbital plane of the merger. From top to bottom: mass distribution log $\Sigma, V$, and $\sigma$. The CALIFA-like hexagonal field of view for the assumed distance is overplotted on the mock images of the first row.


Fig. 5. Left: pixel values within $r_{\mathrm{hm}}$ of $h_{3}$ (upper panel), $h_{4}$ (bottom panel) versus $V / \sigma$ extracted from the simulated kinematic maps for all the simulated remnants M0-M3. Different colors correspond to the different $B / T$ ratios of their progenitors. Right: same as the left panel for one of the CALIFA prolate-rotating galaxies, LSBCF560-04, that shows weak evidence for an $h_{3}-V / \sigma$ correlation.

$\sim 150 \mathrm{~km} \mathrm{~s}^{-1}$, although it contributes only $\sim 20 \%$ to the total stellar mass of the NGC 4365 .

In our simulations, stars that account for the prolate rotation retain the memory of their initial orientation before the merger and for many Gyr of evolution after its progenitors have merged. These stars, which initially belonged to the "polar" progenitor's disk, show a similar amplitude of rotation in the remnants in all simulation setups, hence their mass fraction defines the amplitude of prolate rotation of the whole galaxy.

Figure 6 also shows that the aligned progenitor composes a very elongated almost bar-like component in the final remnant, while the polar progenitor shows a less prolate shape. We find that this is true for all simulation setups, and for example, for simulation $\mathrm{M} 0$, the triaxiality parameter for the aligned progenitor is $T=0.87$, while for the polar $T=0.55$ in the final merger remnant.

The different shapes of the two progenitors in the final merger remnant is a consequence of the different effect of their mutual tidal interactions on their morphology during their merger. Figure 7 shows the two progenitors in simulation M0 in their initial setup before the merger simulation starts $(t=0 \mathrm{Gyr})$ and at their first apocenter $(t=1.05 \mathrm{Gyr})$. While the two galaxies 



Fig. 6. Same as Fig. 4 for the remnants M0 (top two rows) and M3 (bottom two rows). From top to bottom: mass distribution log $\Sigma$ and mock line-of-sight velocity maps for different stellar populations in the remnant. From left to right: the first column shows all the stars of the merger remnant ("All stars"), the second column shows only the particles that initially belonged to the disk of the progenitor that was inclined by $90^{\circ}$ with respect to the orbital plane ("Polar Pr. disk") and show strong prolate rotation in the remnant. The third column shows the same for the particles that initially formed the disk of the progenitor that was aligned with the orbital plane ("Aligned Pr. disk"), while the last column shows the remaining stellar particles of the remnant that initially formed the progenitor bulges ("Pr. bulges"). The mass fraction of each component with respect to the total stellar mass of the remnant is denoted in the top right corner.

are initially almost identical, the aligned progenitor develops a strongly barred morphology soon after the first pericentric passage (top right panel of Fig. 7). This is not the case for the polar progenitor, which retains its disky shape, although it is substantially disturbed (bottom left panel of Fig. 7).

Such tidally induced bars have previously been studied in detail to form after first pericentric passages of disk galaxies during encounters with a perturber. They are long-lived, and are caused by angular momentum transfer from the stellar component of the galaxy to its halo (e.g., Gerin et al. 1990; Aguerri \& González-García 2009; Łokas et al. 2014a, 2016; Martinez-Valpuesta et al. 2017). In our case, the tidally induced bar of the aligned progenitor is also long-lived and survives even after the coalescence of the two galaxies at $t \sim 2 \mathrm{Gyr}$, and until the final time-step of the simulation ( $\sim 6 \mathrm{Gyr}$ after coalescence), the aligned progenitor retains this bar-like prolate shape in the final remnant.

We conclude that in the final merger remnant, the aligned progenitor retains a memory of its shape before coalescence and is mainly responsible for the prolate shape of the remnant. On the other hand, the polar progenitor retains a memory of its angular momentum before coalescence and is responsible for the prolate rotation of the remnant.

We expect that our findings, combined with future orbitbased dynamical models for the CALIFA sample of prolate rotators, can help toward better constraining their dynamical structure as well as their formation history, as the mass fraction of their prolate-rotating populations may provide important information on their assembly process and the nature of their progenitor galaxies.

\section{Summary and discussion}

We presented evidence for 10 ETGs from the CALIFA Survey that show prolate rotation (rotation around the major photometric axis) in their stellar kinematics. This sample includes the discovery of 8 new prolate rotators, adding a significant fraction to the $(\sim 12)$ such cases of massive ETGs that have been reported in the literature so far. We additionally investigated their possible merger origin by studying the stellar kinematics of elliptical merger remnants using $N$-body simulations of major polar galaxy mergers. Our results are summarized below.

(i) Most of the ten prolate rotating galaxies presented here appear to belong to galaxy groups or clusters. Five of them are brightest cluster galaxies (BCGs). Two of them show distinct minor-axis dust lanes (NGC 0810 and NGC 5485). Together with the main stellar body prolate rotation, minoraxis dust lanes are an additional evidence for triaxiality (Bertola \& Galletta 1978; Merritt \& de Zeeuw 1983). We 

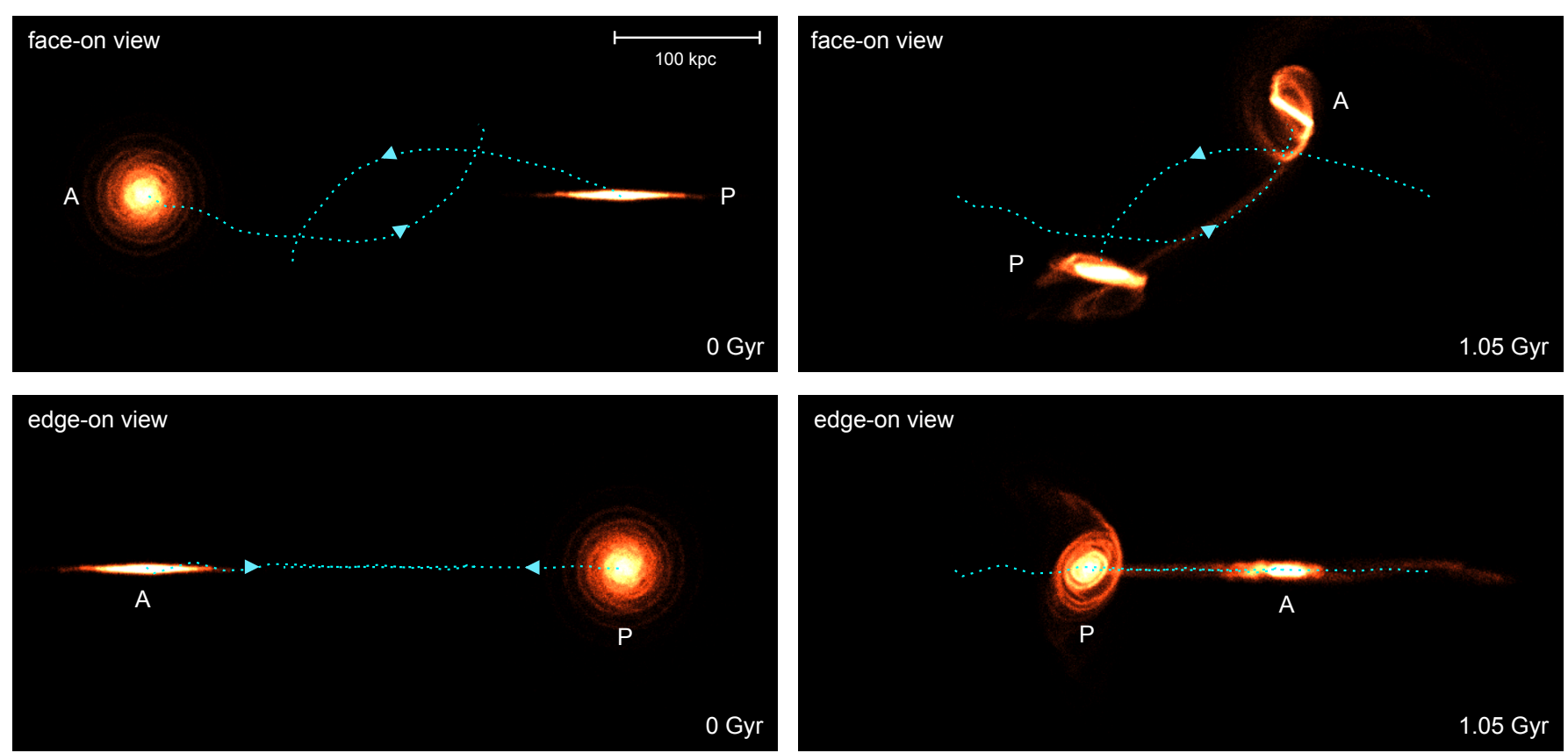

Fig. 7. Face-on (top panels) and edge-on (bottom panels) projection of the orbital plane of the merger simulation M0. The aligned progenitor is denoted "A", while the polar progenitor is denoted "P". The panels on the left show the initial setup before the merger simulation starts $(t=0$ Gyr). The panels on the right show the two progenitors 1.05 Gyr later, at their first apocenter, where the aligned progenitor A has developed a strong tidally induced bar (seen face-on in the top right panel), while this is not the case for the polar progenitor P (seen face-on in the bottom right panel). The merger trajectories of the two progenitors are overplotted. Images were created with glnemo $2^{2}$.

suggest that the galaxies presented here are intrinsically triaxial systems.

(ii) Early-type galaxies with prolate rotation might be more common than previously thought. We have detected 6 prolate rotators out of 81 ETGs in the CALIFA Kinematic Subsample of 300 galaxies as described in Falcón-Barroso et al. (2017). This corresponds to a volume-corrected fraction of $\sim 9 \%$ of ETGs with prolate rotation, and $\sim 27 \%$ in the massive $\left(M_{*} \gtrsim 2 \times 10^{11} M_{\odot}\right)$ end. This unprecedentedly high fraction yields potential implications for ETG formation.

(iii) We investigated the merger scenario, according to which the central stellar body of a prolate rotator was formed by a major merger more than $\sim 10 \mathrm{Gyr}$ ago. As for most of the CALIFA galaxies we see no evidence for oblate stellar rotation (rotation around the short axis), we suggest that their merger formation must have been gas poor. We therefore performed a set of $\mathrm{N}$-body simulations of major polar mergers of disk galaxies and investigated the dynamical structure of their resulting remnants. We find that such remnants exhibit highly prolate shapes with strong prolate rotation that depends on the bulge-to-total stellar mass ratio $(B / T)$ of their progenitor galaxies. The higher this ratio, the lower the amplitude of prolate rotation in the resulting ETGs.

(iv) By constructing mock IFU observations of their stellar kinematics, we find that all the simulated merger remnants show a double peak on their line-of-sight (LOS) velocity dispersion profile along the minor axis. As the $B / T$ of the progenitors increases, the double peaks become weaker. We also find a positive correlation between their LOS velocity and the higher order moment $h_{3}$. This is in contrast with what is observed for most ETGs with oblate rotation (e.g., Krajnović et al. 2008). Better quality observations are now

\footnotetext{
http://projets.lam.fr/projects/glnemo2
}

needed in order to confirm the presence of such kinematic features in the observed prolate rotators.

(v) We show that the prolate rotation in each simulated merger remnant originates from the progenitor galaxy whose disk had an orthogonal orientation with respect to the orbital plane before the merger, while the prolate shape of the remnant originates mainly from the progenitor whose disk was aligned with the orbital plane. The latter is a consequence of the aligned progenitor developing a tidally induced bar before coalescence.

We have investigated the polar merger origin of prolate rotating ETGs, using mergers of two identical disk galaxies with orthogonal disk orientations before the merger. We note that such orientations may be infrequent, and we would expect that varying the initial relative inclinations of the two disks would change the resulting kinematics of the remnant.

The origin of prolate rotation has also been studied in simulations of dwarf galaxy mergers in orthogonal disk orientations (Łokas et al. 2014b), which involved progenitors on radial orbits, however, whose disks were inclined by $45^{\circ}$ with respect to their orbital plane. This formation scenario was suggested in order to explain the prolate rotation that was recently observed in the dwarf spheroidal galaxy Andromeda II (Ho et al. 2012), the first dwarf galaxy observed to show such a feature in the form of a stellar stream rotating around its major projected axis (see also Kacharov et al. 2017, for their recent discovery of prolate rotation in another dwarf galaxy). This feature in Andromeda II was interpreted as evidence of a past major merger between two dwarf galaxies (Amorisco et al. 2014).

By studying this merger scenario, Ebrová \& Łokas (2015) found that mergers between disky dwarfs are needed to explain the prolate rotation in the resulting dwarf galaxy remnants, which can be accounted for by a variety of inclinations of the progenitor disks and orbital plane orientations. 
In line with these findings for dwarf galaxies, we here studied the scenario of polar mergers for the case of massive ETGs with prolate rotation, for which we would expect that they may also result from a variety of initial orientations of their progenitors disks, and our fine-tuning of a strictly orthogonal orientation should not be considered as a limitation to their predicted rate of occurrence.

We only investigated the effect of the $B / T$ fraction of each progenitor here, but we note that various other parameters may influence the resulting kinematics of the merger remnant. Such parameters include not only the initial orbital parameters of the merger, but also other structural parameters of the progenitor disks, as well as their relative mass fraction. Such a further investigation remains beyond the scope of the present work, but it seems now crucial for a better understanding of the formation origin of prolate rotators.

In our simulations we found prolate remnant ETGs with a positive correlation between their LOS velocity and the higherorder-moment $h_{3}$. The quality of the CALIFA data is not sufficient to measure the higher order moments reliably in order to compare with findings from our merger simulations. These CALIFA ETGs require further study with higher resolution observations from next-generation IFU instruments (e.g., MUSE, Bacon et al. 2010) in order to assess the implications of our findings in more detail.

This work, combined with future higher quality observations of the sample of galaxies presented and orbit-based dynamical modeling, will give better insights into the dynamical nature of this special type of rotators. Finally, our findings, combined with results from cosmological simulations, will help to place more accurate constraints on the formation origin and the occurrence rate of prolate rotation in massive ETGs.

Acknowledgements. We are grateful to the anonymous referee for the constructive suggestions made to improve our work, as well as to Hector Hiss, Ivana Ebrová, Davor Krajnović, Eric Emsellem, and Sebastián Sánchez for useful discussions and contributions. This study makes use of the data provided by the Calar Alto Legacy Integral Field Area (CALIFA) survey http://califa. caha.es. Based on observations collected at the Centro Astronómico Hispano Alemán (CAHA) at Calar Alto, operated jointly by the Max-Planck-Institu für Astronomie and the Instituto de Astrofísica de Andalucía (CSIC). J.A.L.A was funded from the grant AYA2013-43188-P, and J.F.B. from grant AYA2016 77237-C3-1-P, by the Ministerio de Economia y Competitividad (MINECO) A.T. and G.v.d.V. acknowledge financial support from the DAGAL network from the People Programme (Marie Curie Actions) of the European Union's Seventh Framework Programme FP7/2007-2013/ under REA grant agreement number PITN-GA-2011-289313. The numerical simulations used in this work were performed on the THEO cluster of the Max-Planck-Institut für Astronomie at the Rechenzentrum in Garching. Glnemo2 copyright: Jean-Charles Lambert. Glnemo2 was developed at CeSAM/LAM.

\section{References}

Aarseth, S. J., \& Binney, J. 1978, MNRAS, 185, 227

Abazajian, K. N., Adelman-McCarthy, J. K., Agüeros, M. A., et al. 2009, ApJS, 182,543

Aguerri, J. A. L., \& González-García, A. C. 2009, A\&A, 494, 891

Amorisco, N. C., Evans, N. W., \& van de Ven, G. 2014, Nature, 507, 335

Arnold, J. A., Romanowsky, A. J., Brodie, J. P., et al. 2014, ApJ, 791, 80

Bacon, R., Accardo, M., Adjali, L., et al. 2010, in Ground-based and Airborne Instrumentation for Astronomy III, Proc. SPIE, 7735, 773508

Bell, E. F., Wolf, C., Meisenheimer, K., et al. 2004, ApJ, 608, 752

Bendo, G. J., \& Barnes, J. E. 2000, MNRAS, 316, 315

Bertola, F., \& Galletta, G. 1978, ApJ, 226, L115

Bertola, F., Galletta, G., Capaccioli, M., \& Rampazzo, R. 1988, A\&A, 192, 24 Binney, J. 1985, MNRAS, 212, 767

Bois, M., Emsellem, E., Bournaud, F., et al. 2011, MNRAS, 416, 1654
Burke, C., \& Collins, C. A. 2013, MNRAS, 434, 2856 Cappellari, M., \& Copin, Y. 2003, MNRAS, 342, 345 Carter, D., Thomson, R. C., \& Hau, G. K. T. 1998, MNRAS, 294, 182 Chang, J., Macciò, A. V., \& Kang, X. 2013, MNRAS, 431, 3533 Contopoulos, G. 1956, ZAp, 39, 126

Cox, T. J., Dutta, S. N., Di Matteo, T., et al. 2006, ApJ, 650, 791

Davies, R. L., \& Birkinshaw, M. 1986, ApJ, 303, L45

Davies, R. L., \& Birkinshaw, M. 1988, ApJS, 68, 409

Davies, R. L., \& Illingworth, G. D. 1986, ApJ, 302, 234

Davies, R. L., Kuntschner, H., Emsellem, E., et al. 2001, ApJ, 548, L33

Deibel, A. T., Valluri, M., \& Merritt, D. 2011, ApJ, 728, 128

Ebrová, I., \& Łokas, E. L. 2015, ApJ, 813, 10

Emsellem, E., Cappellari, M., Peletier, R. F., et al. 2004, MNRAS, 352, 721

Emsellem, E., Cappellari, M., Krajnović, D., et al. 2011, MNRAS, 414, 888

Emsellem, E., Krajnović, D., \& Sarzi, M. 2014, MNRAS, 445, L79

Faber, S. M., Willmer, C. N. A., Wolf, C., et al. 2007, ApJ, 665, 265

Falcón-Barroso, J., Lyubenova, M., van de Ven, G., et al. 2017, A\&A, 597, A48

Foster, C., Arnold, J. A., Forbes, D. A., et al. 2013, MNRAS, 435, 3587

Franx, M., Illingworth, G., \& de Zeeuw, T. 1991, ApJ, 383, 112

Gerin, M., Combes, F., \& Athanassoula, E. 1990, A\&A, 230, 37

Heisler, J., Merritt, D., \& Schwarzschild, M. 1982, ApJ, 258, 490

Hernquist, L. 1990, ApJ, 356, 359

Ho, N., Geha, M., Munoz, R. R., et al. 2012, ApJ, 758, 124

Hoffman, L., Cox, T. J., Dutta, S., \& Hernquist, L. 2010, ApJ, 723, 818

Jedrzejewski, R., \& Schechter, P. L. 1989, AJ, 98, 147

Jesseit, R., Naab, T., Peletier, R. F., \& Burkert, A. 2007, MNRAS, 376, 997

Johansson, P. H., Naab, T., \& Ostriker, J. P. 2012, ApJ, 754, 115

Kacharov, N., Battaglia, G., Rejkuba, M., et al. 2017, MNRAS, 466, 2006

Kelz, A., Verheijen, M. A. W., Roth, M. M., et al. 2006, PASP, 118, 129

Khochfar, S., \& Silk, J. 2009, MNRAS, 397, 506

Krajnović, D., Cappellari, M., de Zeeuw, P. T., \& Copin, Y. 2006, MNRAS, 366, 787

Krajnović, D., Bacon, R., Cappellari, M., et al. 2008, MNRAS, 390, 93

Krajnović, D., Emsellem, E., Cappellari, M., et al. 2011, MNRAS, 414, 2923

Kutdemir, E., Ziegler, B. L., Peletier, R. F., et al. 2008, A\&A, 488, 117

Lackner, C. N., Cen, R., Ostriker, J. P., \& Joung, M. R. 2012, MNRAS, 425, 641

Łokas, E. L., Athanassoula, E., Debattista, V. P., et al. 2014a, MNRAS, 445, 1339

Łokas, E. L., Ebrová, I., Pino, A. d., \& Semczuk, M. 2014b, MNRAS, 445, L6 Łokas, E. L., Ebrová, I., del Pino, A., et al. 2016, ApJ, 826, 227

Martinez-Valpuesta, I., Aguerri, J. A. L., González-García, A. C., Dalla Vecchia, C., \& Stringer, M. 2017, MNRAS, 464, 1502

McDermid, R. M., Emsellem, E., Shapiro, K. L., et al. 2006, MNRAS, 373, 906

Merritt, D., \& de Zeeuw, T. 1983, ApJ, 267, L19

Moellenhoff, C., \& Bender, R. 1989, A\&A, 214, 61

Moellenhoff, C., \& Marenbach, G. 1986, A\&A, 154, 219

Naab, T., \& Burkert, A. 2003, ApJ, 597, 893

Naab, T., Oser, L., Emsellem, E., et al. 2014, MNRAS, 444, 3357

Norris, M. A., Van de Ven, G., Schinnerer, E., et al. 2016, ApJ, 832, 198

Pérez, E., Cid Fernandes, R., González Delgado, R. M., et al. 2013, ApJ, 764, L1

Roth, M. M., Kelz, A., Fechner, T., et al. 2005, PASP, 117, 620

Sánchez, S. F., Kennicutt, R. C., Gil de Paz, A., et al. 2012, A\&A, 538, A8

Sánchez, S. F., García-Benito, R., Zibetti, S., et al. 2016, A\&A, 594, A36

Schechter, P. L., \& Gunn, J. E. 1979, ApJ, 229, 472

Springel, V. 2005, MNRAS, 364, 1105

Springel, V., Di Matteo, T., \& Hernquist, L. 2005, MNRAS, 361, 776

Statler, T. S., Emsellem, E., Peletier, R. F., \& Bacon, R. 2004, MNRAS, 353, 1

Tsatsi, A., Macciò, A. V., van de Ven, G., \& Moster, B. P. 2015, ApJ, 802, L3

Valdes, F., Gupta, R., Rose, J. A., Singh, H. P., \& Bell, D. J. 2004, ApJS, 152, 251

van Albada, T. S., Kotanyi, C. G., \& Schwarzschild, M. 1982, MNRAS, 198, 303 van den Bosch, R. C. E., van de Ven, G., Verolme, E. K., Cappellari, M., \& de Zeeuw, P. T. 2008, MNRAS, 385, 647

van der Marel, R. P., \& Franx, M. 1993, ApJ, 407, 525

van der Wel, A., Franx, M., van Dokkum, P. G., et al. 2014, ApJ, 788, 28

van de Ven, G., van den Bosch, R. C. E., Verolme, E. K., \& de Zeeuw, P. T. 2006,

A\&A, 445, 513

van Dokkum, P. G., Franx, M., Kriek, M., et al. 2008, ApJ, 677, L5

van Dokkum, P. G., Whitaker, K. E., Brammer, G., et al. 2010, ApJ, 709, 1018

Verheijen, M. A. W., Bershady, M. A., Andersen, D. R., et al. 2004, Astron.

Nachr., 325, 151

Vitvitska, M., Klypin, A. A., Kravtsov, A. V., et al. 2002, ApJ, 581, 799

Wagner, S. J., Bender, R., \& Moellenhoff, C. 1988, A\&A, 195, L5

Walcher, C. J., Wisotzki, L., Bekeraité, S., et al. 2014, A\&A, 569, A1

Wilkinson, A., \& James, R. A. 1982, MNRAS, 199, 171

Zirm, A. W., van der Wel, A., Franx, M., et al. 2007, ApJ, 656, 66 